\title{
COMMENTARY
}

\section{Treatment of candidemia in adult patients without neutropenia - an inconvenient truth}

\author{
Pedro Póvoa* and João Gonçalves-Pereira
}

\begin{abstract}
In 2009 the Infectious Diseases Society of America reviewed the guidelines on the treatment of candidemia in non-neutropenic patients. In this document the preferred treatment was either fluconazole or an echinocandin. Amphotericin-B formulations were considered an alternative. However, careful assessment of published data showed similar efficacy between these drugs.
\end{abstract}

\section{Introduction}

Fungal infections, in particular candidemia, are a growing problem in immunocompetent patients [1]. Despite several guidelines, concern exists on the value of evidence supporting recommendations for the optimal treatment of candidemia in non-neutropenic adult patients [1,2].

In the midst of this controversy a critical reappraisal of the 2009 Infectious Diseases Society of America (IDSA) guidelines is appropriate [1]. Although many relevant aspects of the guidelines are thoroughly discussed, there are three topics that deserve a closer look: the weight and quality of the evidence; the evidence of efficacy; and the evidence of amphotericin-B (AmB)-induced renal failure.

\section{What is recommended by the 2009 IDSA guidelines?}

In 2009, IDSA updated their 2004 guidelines (Table 1) $[1,2]$. The primary recommendation for treatment of candidemia in non-neutropenic patients was administration of fluconazole or an echinocandin. The alternative approach was administration of lipid formulation (LF)AmB, AmB-deoxycholate (AmB-d) or voriconazole. The recommendation for echinocandins was extended to patients with moderately severe to severe illness or with recent azole exposure.

*Correspondence: povoap@netcabo.pt

Unidade de Cuidados Intensivos Polivalente, Hospital São Francisco Xavier, Centro Hospitalar de Lisboa Ocidental, EPE, Estrada do Forte do Alto do Duque, 1449-005 Lisboa, Portugal

\section{A critical review of the evidence}

The 2009 IDSA guidelines [1] represent a marked change in the recommended therapeutic approach, and is not supported by the efficacy of the different antifungals. In all nine clinical trials, AmB-d and LF-AmB were never inferior to any comparator and two recent meta-analyses showed the same results [3,4]. The use of AmB-d and LF$A m B$ are supported by very solid data from well designed clinical trials and that have consequently been twice graded A-I [1]. Moreover, echinocandins are not suitable for the treatment of endophtalmitis, meningitis and endocarditis [5] whilst AmB remains the drug of choice.

Therefore, even though not explicit in the guidelines, the change is probably related to a possible advantage of echinochandins and the perceived renal dysfunction associated with AmB. In fact, the 2009 IDSA guidelines [1] attribute to AmB-d therapy a high risk of acute renal failure (ARF) and mortality.

\section{The new evidence}

A recent study from Reboli and colleagues [6] suggests that anidulafungin might be superior to fluconazole as primary therapy for candidemia in non-neutropenic patients $(75.6 \%$ success with anidulafungin compared to $60.2 \%$ with fluconazole). However, in a noninferiority trial, only noninferiority can be demonstrated and any benefit of the new treatment should be interpreted with great caution [7] and only be based on other advantages, such as safety, convenience and cost [8]. Besides, the evaluation of the primary end-point was made at the end of intravenous therapy, after a median of 14 days of anidulafungin against only 11 days of fluconazole. In addition, more patients in the anidulafungin group had their central venous catheter removed than in the fluconazole arm (96\% versus $89 \%$ ). Consequently, as Sobel and Revankar wrote in an editorial [9], anidulafungin was, at best, noninferior in comparison to fluconazole.

\section{The myths on nephrotoxicity}

The change of the positioning of $\mathrm{AmB}$ preparations in the 2009 guidelines [1,2] seems to be supported by the nephrotoxicity findings of two studies, one showing that 
Table 1. Infectious Disease Society of America guidelines for the treatment of candidemia in non-neutropenic adult patients [1,2]

\begin{tabular}{|c|c|c|}
\hline & 2004 & 2009 \\
\hline Recommended therapy & $\begin{array}{l}\text { AmB-d } 0.6 \text { to } 1 \text { mg/kg/day } \\
\text { Fluconazole } 400 \text { to } 800 \text { mg/day } \\
\text { Caspofungin } 50 \text { mg/day (70 mg first dose) }\end{array}$ & $\begin{array}{l}\text { Echinochandina: Caspofungin } 50 \text { mg/day (70 mg first dose) } \\
\text { Anidulafungin } 100 \text { mg/day (200 mg first dose) } \\
\text { Micafungin } 100 \text { mg/day } \\
\text { Fluconazol } 6 \text { mg/kg/day (12 mg/kg first dose) }\end{array}$ \\
\hline Alternative therapy & $\begin{array}{l}\text { AmB-d } 0.7 \text { mg/kg/day + Fluconazol } 800 \text { mg/day } \\
\text { ( } 4 \text { to } 7 \text { days, then Fluconazol } 800 \text { mg/day) }\end{array}$ & $\begin{array}{l}\text { LF-AmB } 3 \text { to } 5 \text { mg/kg/day } \\
\text { AmB-d } 0.5 \text { to } 1 \mathrm{mg} / \mathrm{kg} \\
\text { Voriconazol } 3 \mathrm{mg} / \mathrm{kg} \text { bid ( } 6 \mathrm{mg} / \mathrm{kg} \text { first two doses) }\end{array}$ \\
\hline Duration of therapy & At least 14 days after last positive blood culture & At least 14 days after last positive blood culture \\
\hline Other recommendations & $\begin{array}{l}\text { Removal of central venous catheter } \\
\text { Ophthalmological evaluation }\end{array}$ & $\begin{array}{l}\text { Removal of central venous catheter } \\
\text { Ophthalmological evaluation }\end{array}$ \\
\hline
\end{tabular}

${ }^{\mathrm{a}} \mathrm{An}$ echinochandin was also recommended in moderate to severe infection or with recent azole exposure. AmB-d, Amphotericin-B deoxycholate; LF-AmB, lipid formulation Amphotericin-B.

Table 2. Amphotericin-B nephrotoxicity; data cited in 2009 Infectious Disease Society of America guidelines [1]

\begin{tabular}{lll}
\hline & Wingard et al. [12] & Bates et al. [11] \\
\hline Year of publication & 1999 & 2001 \\
Data collection & Jan 1991 to Dec 1993 & May 1993 to Apr 1997 \\
Inclusion criteria & 1. Allogeneic BMT & Not patients but number of admissions; at least \\
& 2. Autologous BMT & 156 repeated measurements; major violation of the \\
& 4. Solid organ transplantation & assumptions of statistical methods used \\
& transplantation & \\
Infection & Aspergillosis & Unknown \\
Duration of AmB (days) & 20.4 & 14.8 \\
AmB cumulative dose (g) & Unknown & 0.93 \\
AmB daily dose (mg/kg) & Unknown & Unknown \\
Concomitant nephrotoxic agents & $87 \%$ & Collected (not given) \\
Risk of death & HD $>3 \times$ & ARF $>6.6 \times$
\end{tabular}

$A m B, A m p h o t e r i c i n-B ; A R F$, acute renal failure defined as a $50 \%$ increase in the baseline creatinine level, with a peak of $>2.0 \mathrm{mg} / \mathrm{dL}$; $B M T$, bone marrow transplant; $H D$, haemodialysis.

AmB-d could result in ARF in up to $50 \%$ of patients [10] and the other that AmB-d-induced nephrotoxicity is associated with a 6.6-fold increased risk of death [11] (Table 2).

The first study is a review about AmB nephrotoxicity [10]; in this review, the author cited another study published in 1999 [12] where those findings were presented. However, patients included in this retrospective analysis were markedly immunosupressed, received concomitantly nephrotoxic agents, and received long courses of AmB (20.4 days) for suspected or proven aspergillosis, not invasive candidiasis. Accordingly, these findings cannot be extrapolated to non-neutropenic patients with candidemia.

The second study [11] is also a retrospective analysis published in 2001, using data from 707 admissions of 551 patients treated with AmB-d. However, the inclusion of the same patient several times constitutes a major violation of the assumptions necessary to compare groups with the statistical methods used. Roughly, 30\% of these admissions were complicated with ARF. The average highest creatinine in this group was $3.3 \pm 1.3 \mathrm{mg} / \mathrm{dL}$, whereas in those without ARF it was $1.6 \pm 0.7 \mathrm{mg} / \mathrm{dL}$ $(P<0.0001)$. The mortality rate in the admissions complicated with ARF was 54.2\% and in those without ARF it was only $16 \%$ (odds ratio for death, 6.6). From these data, it is difficult to envisage that patients with a mean increase in creatinine of $1.7 \mathrm{mg} / \mathrm{dL}$ had a 6.6 -fold increased risk of death.

Besides, as we have already pointed out, AmB was never inferior to any comparator in six randomised controlled trials with non-neutropenic adult patients with candidemia [3,4]. If AmB-induced nephrotoxicity was so common and has such a detrimental effect on prognosis, it would be expected that this finding would have a negative effect on mortality.

\section{Should we still use AmB-d?}

Even though AmB-d is an old drug, it is still regarded as one of the drugs of choice for treatment of 
life-threatening mycoses as well as for the empirical therapy of febrile neutropenia. A Cochrane review published in 2000, but left unchanged after reassessments in 2007 and in 2009, provides support for this practice [13]. That is probably because AmB-d remains the antifungal with the most rapid time-kill rate and the largest post-antifungal effect [14] and with efficacy that increases with its concentration [15]. LF-AmB could present a better safety profile, but its cost constitutes a major limitation to its routine use. In addition, there are no solid data to support any benefit from LF-AmB in comparison to AmB-d if administered with correct pre-medication.

\section{Conclusion}

Careful assessment of published data on the treatment of candidemia in non-neutropenic patients showed similar efficacy between $\mathrm{AmB}$ preparations, fluconazole and echinochandins. The choice of first line therapy should be based on individual risk factors, patterns of Candida susceptibility, clinical experience, as well as local availability of the different drugs and their costs.

\section{Abbreviations}

$A m B$, amphotericin-B; AmB-d, amphotericin-B deoxycholate; ARF, acute renal failure; IDSA, Infectious Diseases Society of America; LF-AmB, lipid formulation of amphotericin-B.

\section{Competing interests}

PP has received honoraria and served as advisor of Astra Zeneca, Ely-Lilly, Gilead, Janssen-Cilag, Merck Sharp and Dohme, Novartis and Pfizer. JGP has received honoraria and served as advisor of Pfizer, Astra-Zeneca, Abbott, Wyeth-Lederle, Janssen-Cilag, Merck Sharp and Dohme, and received an unrestricted research grant from Astra-Zeneca.

\section{Authors' contributions}

PP and JGP contributed to the conception, analysis and interpretation of data of the present commentary and were involved in drafting the manuscript and its revision.

\section{Authors' information}

PP is coordinator of the Polyvalent Intensive Care Unit and president of the Antibiotic Commission of São Francisco Xavier Hospital. PP is Professor of Medicine of the Faculty of Medical Sciences from the New University of Lisbon, Portugal. JGP is consultant in Polyvalent Intensive Care Unit whose main field of interest is infection and antibiotic chemotherapy.

\section{Acknowledgements}

We would like to express our gratitude to Prof. Jorge Salluh for critical reading of this manuscript. This commentary was supported by an unrestricted grant from Gilead.
Published: 31 January 2011

\section{References}

1. Pappas PG, Kauffman CA, Andes D, Benjamin DK Jr, Calandra TF, Edwards JE Jr, Filler SG, Fisher JF, Kullberg BJ, Ostrosky-Zeichner L, Reboli AC, Rex JH, Walsh TJ, Sobel JD: Clinical practice guidelines for the management of candidiasis: 2009 update by the Infectious Diseases Society of America. Clin Infect Dis 2009, 48:503-535.

2. Pappas PG, Rex JH, Sobel JD, Filler SG, Dismukes WE, Walsh TJ, Edwards JE: Guidelines for treatment of candidiasis. Clin Infect Dis 2004, 38:161-189.

3. Gafter-Gvili A, Vidal L, Goldberg E, Leibovici L, Paul M: Treatment of invasive candidal infections: systematic review and meta-analysis. Mayo Clin Proc 2008, 83:1011-1021.

4. Mills EJ, Perri D, Cooper C, Nachega JB, Wu P, Tleyjeh I, Phillips P: Antifungal treatment for invasive Candida infections: a mixed treatment comparison meta-analysis. Ann Clin Microbiol Antimicrob 2009, 8:23.

5. Bennett JE: Echinocandins for candidemia in adults without neutropenia. NEngl J Med 2006, 355:1154-1159.

6. Reboli AC, Rotstein C, Pappas PG, Chapman SW, Kett DH, Kumar D, Betts R, Wible M, Goldstein BP, Schranz J, Krause DS, Walsh TJ: Anidulafungin versus fluconazole for invasive candidiasis. N Engl J Med 2007, 356:2472-2482.

7. Gotzsche PC: Lessons from and cautions about noninferiority and equivalence randomized trials. JAMA 2006, 295:1172-1174.

8. Kaul S, Diamond GA: Making sense of noninferiority: a clinical and statistical perspective on its application to cardiovascular clinical trials. Prog Cardiovasc Dis 2007, 49:284-299.

9. Sobel JD, Revankar SG: Echinocandins - first-choice or first-line therapy for invasive candidiasis? N Engl J Med 2007, 356:2525-2526.

10. Deray G: Amphotericin B nephrotoxicity. J Antimicrob Chemother 2002, 49 Suppl 1:37-41.

11. Bates DW, Su L, Yu DT, Chertow GM, Seger DL, Gomes DR, Dasbach EJ, Platt R: Mortality and costs of acute renal failure associated with amphotericin B therapy. Clin Infect Dis 2001, 32:686-693.

12. Wingard JR, Kubilis P, Lee L, Yee G, White M, Walshe L, Bowden R, Anaissie E, Hiemenz J, Lister J: Clinical significance of nephrotoxicity in patients treated with amphotericin B for suspected or proven aspergillosis. Clin Infect Dis 1999, 29:1402-1407.

13. Johansen HK, Gøtzsche PC: Amphotericin B lipid soluble formulations versus amphotericin $B$ in cancer patients with neutropenia. Cochrane Database Systematic Rev 2000:CD000969.

14. Di Bonaventura G, Spedicato I, Picciani C, D'Antonio D, Piccolomini R: In vitro pharmacodynamic characteristics of amphotericin B, caspofungin, fluconazole, and voriconazole against bloodstream isolates of infrequent Candida species from patients with hematologic malignancies. Antimicrob Agents Chemother 2004, 48:4453-4456.

15. Canton E, Peman J, Gobernado M, Viudes A, Espinel-Ingroff A: Patterns of amphotericin B killing kinetics against seven Candida species. Antimicrob Agents Chemother 2004, 48:2477-2482.

doi:10.1186/cc9414

Cite this article as: Póvoa P, Gonçalves-Pereira J: Treatment of candidemia in adult patients without neutropenia - an inconvenient truth. Critical Care 2011, 15:114. 